\title{
Prediction of acute pancreatitis severity via the combined analysis of inflammatory biomarkers and coagulation parameters
}

\author{
Snezana Tesic Rajkovic ${ }^{1 *}$, Biljana Radovanovic Dinic ${ }^{2}$, Miodrag Djordjevic ${ }^{3}$, \\ Goran Marjanovic ${ }^{4}$, Sasa Grgov ${ }^{5}$ \\ 1. Clinic for Gastroenterology and Hepatology, Clinical Centre Nis, Serbia \\ 2. Clinic for Gastroenterology and Hepatology, Clinical Centre, Faculty of Medicine, Nis, Serbia \\ 3. Faculty of Sciences and Mathematics, Nis, Serbia \\ 4. Clinic for Hematology, Clinical Centre, Faculty of Medicine, Nis, Serbia \\ 5. General Hospital, Leskovac, Serbia
}

\begin{abstract}
Introduction. Timely assessment of severity of acute pancreatitis is needed to avoid severe systemic complications by making optimal therapeutic approach and correct prognosis of the disease. The aim of the study was to establish the role of several inflammatory biomarkers and coagulation parameters in prediction of AP severity, and also to propose a mathematical formula which allows their combined use for the same purpose.

Material and Methods. The prospective study included 70 patients with AP. The patients were divided into groups: mild (group I), moderate (group II) and severe AP (group III). All patients were further classified into two groups: group A (mild AP) and group B (moderate and severe AP). Biochemical markers, inflammatory biomarkers and coagulation factors were tested in all patients.

Results. Based on the results of Mann-Whitney,s test, it can be concluded that groups A and B are significant different from each other for CRP $(p<0.05)$. Using the Wald's stepwise forward method, a prediction model with CRP, PCT, D-dimer1, D-dimer3, fibrinogen1 and fibrinogen3 parameters as predictors of the severity of AP was obtained. The percentage of successful prediction of moderate or severe AP based on this model was 76.9\%. The use of ROC analysis with the introduced linear combination from the logistic regression yielded equally good or even better results in the assessment of the severity of AP with the combined use of analyzed parameters.

Conclusion. The combined analyses of biohumoral markers and coagulation parameters presented in the form a mathematical formula enabled a more accurate, rapid, rational and clinically available prediction of the severity of $A P$.
\end{abstract}

Keywords: acute pancreatitis, procalcitonin, $C$ reactive protein, $D$ dimer

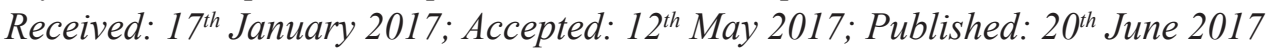

*Corresponding author: Snezana Tesic Rajkovic, Clinic for Gastroenterology and Hepatology, Clinical Centre Nis, 18000 Nis, Serbia, e-mail: snezanatesic@yahoo.com 


\section{Introduction}

Acute pancreatitis (AP) is an inflammatory disease of the pancreas with different morphological and clinical features, unpredictable course, and uncertain outcome. Mediators of inflammation can come into the systemic circulation and lead to the development of systemic inflammatory response syndrome (SIRS) with consequent multiple organ dysfunction syndrome (MODS).

In the evolution of AP, initial changes occur at the level of pancreatic cells where pancreatic enzymes are prematurely activated, which in turn causes vasoconstriction, capillary stasis, reduced oxygen saturation, increased capsular permeability and consequent pancreatic edema [1]. During the further process, leucocytes migrate to the damaged tissue and release proinflammatory cytokines, arachidonic acid metabolites, proteolytic and lipolytic enzymes. The process of inflammation in AP is mutually connected with the coagulation system. The connection between inflammation and the coagulation system in AP is facilitated by specific cell receptors, protease-activated receptors (PAR) that are present on the inflammatory and endothelial cells. Activated coagulation proteases may induce signals and thus modify the inflammatory response by binding to these receptors [2]. The coagulation alterations in pancreatitis may have prognostic values, and increasing data from animal studies proposed that inhibition of coagulation has beneficial effects on disease severity [3]. Studies on the clinical significance of parameters of the coagulation system in predicting complications caused by pancreatitis are rare [4-6].

The widely accepted revised Atlanta classification is a useful framework for assessing the severity of AP. The current classification recognizes three levels of severity: mild, where patients recover with good supportive care within a week without complication; moderate, with transient organ failure lasting up to 48 hours or complications that include acute fluid collections or the exacerbation of underlying chronic disease; and severe AP, with persistent organ failure for more than 48 hours [7].

The early evaluation of AP is key for affected patients, since it allows for a timely introduction of intensive therapy, endoscopic sphincterotomy for severe biliary AP, and necrectomy in infected pancreatic necrosis and peripancreatic tissue. Timely treatment contributes to the prevention of progressive processes that lead to MODS, which is the greatest cause of death in patients with AP. Thus, there are clear intentions to identify prognostic parameters, both individual and grouped, which could provide a more accurate prediction of the outcome of AP. To this purpose, a variety of clinical parameters, scoring systems, laboratory markers and radiological procedures are used. Today, a large number of multifactorial scoring systems like Ranson, Glasgow, APACHE II, and Sequential Organ Failure Assessment (SOFA) score are used to assess the severity of AP. They mainly require the determination of various parameters, with some of them also requiring a specific time, and others being very complicated for calculation [8-10].

The severity of AP may present with clinical signs such as muscle defans, palpable resistance in the abdomen, fever and shock with MODS. However, at the time at which these signs and symptoms appear it is often too late to react, therefore they are of limited predictive value.

In the last 50 years, much effort has been devoted to researching laboratory parameters which can help divide patients with AP into clear risk groups for the development of complications and death. The ideal laboratory test for evaluating the severity and prognosis of AP should be simple, routinely and quickly available in urgent cases, accurate and inexpensive [11]. Today, unique, efficient and inexpensive system or pa- 
rameter with a high specificity and sensitivity for the prediction of AP does not exist.

Taking into account the connections between inflammation and the coagulation system in AP, the individual role of inflammatory biomarkers and coagulation parameters in the evaluation of $\mathrm{AP}$ and their availability in routine practice, as well as the absence of a superior predictive score system, we set ourselves a goal of analyzing the role of certain individual inflammatory biomarkers and coagulation parameters in the prediction of AP severity, and also to propose a mathematical formula which allows their combined use for the same purpose.

\section{Material and Methods}

The prospective study involved 70 patients with the diagnosis of AP who were treated at the Clinic for Gastroenterology and hepatology, Clinical center in Niš during the period from December 2014 to June 2015. The diagnosis of AP was made according to the Atlanta classification which was based on clinical findings that suggested inflammation of the pancreas, including presence of periumbilical or epigastric pain and laboratory confirmation of one or more of the following: serum amylase and/or lipase activity at least three times greater than the upper limit of normal and ultrasonographic or computed tomographic evidence of pancreatic inflammation [12]. The study included only patients whose symptoms started $24 \mathrm{~h}$ before admission. This study did not include pregnant women, patients who received anticoagulation therapy, patients with concomitant severe diseases (the increased risk of thromboembolic complications) or those with chronic liver disease and/or malignancy. Biochemical markers, inflammatory biomarkers (C-reactive protein-CRP, procalcitonin-PCT) and coagulation parameters (prothrombin timePT, partial thromboplastin time-APTT, fibrinogen and D-dimer) were tested in all patients in the Hematology and Biochemical laboratories of the Clinical Center in Niš. Coagulation factors were tested on the first day, and on the third day after admission. In accordance with the World Medical Association Declaration of Helsinki, all the examined patients gave their personal consents related to the use of their blood samples for this research.

The patients were divided into three groups based on the criteria of the revised Atlanta Classification. The group with mild AP (I) included 37 patients. The group with moderate AP (II) consisted of 15 patients and the third group with severe AP (III) comprised 18 patients. The patients with severe AP had commonly persistent systolic blood pressure $<90 \mathrm{~mm} \mathrm{Hg}$ ), pulmonary insufficiency $(\mathrm{PaO} 2<60 \mathrm{~mm} \mathrm{Hg})$ and/or renal failure (creatinine $>300 \mu \mathrm{g} / \mathrm{dl}$ after rehydration). The seriously-ill patients were hospitalized in the intensive care unit of the Clinic for Gastroenterology and Hepatology. The patients with infection of the pancreas or extrapancreatic necrosis (44.44\% from patients with severe AP) were translated in the Clinic for surgery. A lethal outcome occurred in $5(7.1 \%)$ patients with persistent organ failure and development of infected necrosis.

All patients were further classified into two groups: group A (mild AP) with 37 patients, and group B (moderate and severe AP) with 33 patients. The division into A and B group was done with the aim of separating the patients with potentially poor prognosis (group B).

To determine significant differences between groups of patients, t-test for independent samples and Mann-Whitney U-test, depending on normality of data, were used. Binary logistic regression with Enter and Wald's Forward stepwise methods were used for predicting the severity of AP. For testing the diagnostic power ROC analyses were used. All statistical analyses were performed using SPSS 17.0 statistical package. 


\section{Results}

Of the 70 patients ( 37 females and 33 males), mean age was $61.58 \pm 15.42$ years, (from 27 to 77 years) in $28(40.0 \%)$ patients AP had biliary etiology, in $11(15.7 \%)$ alcoholic, and in 31 (44.3\%) patients AP had other etiology.

Table I shows the results of the Mann-Whitney $U$ test used in order to examine differences in average values of parameters done on the first day of hospitalization (PCT, CRP, PT1, APTT1,
INR1, D-dimer1, fibrinogen1) and on the third day of hospitalization (PT3, APTT3, INR3, D-dimer3, fibrinogen3) between group A and group B AP. Parameter with statistically significant difference was CRP $(p<0.05)$.

Prediction of mild, on the one hand (group A), and moderate and severe, on the other (group B) AP based on the first binary logistic regression model was $75.7 \%$ successful. Using the Wald's stepwise forward method, a prediction model with CRP, PCT, D-dimer1, D-dimer3, fi-

Table I. Results of Mann-Whitney $U$ test obtained by comparing parameters of the A and $B$ groups of patients

\begin{tabular}{|c|c|c|c|c|c|c|c|c|}
\hline Parameter & Group & $\mathbf{N}$ & Mean & Std.Dev. & Median & Min & Max & Sig \\
\hline \multirow{2}{*}{ CRP (mg/L) } & $\mathrm{A}$ & 37 & 51.42 & 60.17 & 38.50 & 2.30 & 449.10 & \multirow{2}{*}{0.039} \\
\hline & $\mathrm{B}$ & 32 & 94.21 & 99.33 & 33.40 & 0.80 & 239.00 & \\
\hline \multirow{2}{*}{ PCT (ng/ml) } & A & 31 & 0.45 & 0.76 & 0.18 & 0.02 & 52.88 & \multirow{2}{*}{0.343} \\
\hline & $\mathrm{B}$ & 32 & 3.02 & 10.09 & 0.12 & 0.02 & 3.60 & \\
\hline \multirow{2}{*}{ D-dimer 1 (ng/ml) } & A & 37 & 1025.68 & 1273.98 & 736.00 & 213.00 & 5250.00 & \multirow{2}{*}{0.138} \\
\hline & $\mathrm{B}$ & 33 & 1344.76 & 1476.54 & 582.00 & 159.00 & 5250.00 & \\
\hline \multirow{2}{*}{ D-dimer 3 (ng/ml) } & A & 30 & 762.87 & 901.57 & 777.00 & 185.00 & 5212.00 & \multirow{2}{*}{0.174} \\
\hline & $\mathrm{B}$ & 16 & 1588.56 & 1748.14 & 474.50 & 167.00 & 3681.00 & \\
\hline \multirow{2}{*}{ PT 1 (s) } & $\mathrm{A}$ & 36 & 81.6 & 21.86 & 79.45 & 37.90 & 113.00 & \multirow{2}{*}{0.406} \\
\hline & $\mathrm{B}$ & 30 & 79.78 & 17.65 & 83.60 & 13.40 & 118.00 & \\
\hline \multirow{2}{*}{ PT 3 (s) } & A & 31 & 80 & 20.43 & 80.80 & 56.10 & 136.00 & \multirow{2}{*}{0.643} \\
\hline & $\mathrm{B}$ & 17 & 82.16 & 20.79 & 83.90 & 30.00 & 111.00 & \\
\hline \multirow{2}{*}{ APTT 1 (s) } & A & 37 & 26.79 & 3.89 & 25.40 & 17.10 & 31.70 & \multirow{2}{*}{0.151} \\
\hline & $\mathrm{B}$ & 33 & 25.32 & 3.14 & 25.90 & 20.30 & 39.70 & \\
\hline \multirow{2}{*}{ APTT $3(\mathrm{~s})$} & $\mathrm{A}$ & 31 & 26.72 & 3.85 & 27.40 & 23.40 & 32.50 & \multirow{2}{*}{0.327} \\
\hline & $\mathrm{B}$ & 17 & 27.42 & 2.95 & 26.00 & 21.40 & 36.50 & \\
\hline \multirow{2}{*}{ INR 1} & $\mathrm{~A}$ & 35 & 1.34 & 0.7 & 1.18 & 0.99 & 2.14 & \multirow{2}{*}{0.971} \\
\hline & $\mathrm{B}$ & 33 & 1.23 & 0.23 & 1.18 & 0.92 & 5.00 & \\
\hline \multirow{2}{*}{ INR 3} & $\mathrm{~A}$ & 31 & 1.35 & 0.64 & 1.22 & 0.91 & 1.57 & \multirow{2}{*}{0.666} \\
\hline & $\mathrm{B}$ & 17 & 1.21 & 0.18 & 1.15 & 1.00 & 4.30 & \\
\hline \multirow{2}{*}{ Fibrinogen $1(\mathrm{~g} / \mathrm{L})$} & A & 35 & 5.74 & 1.53 & 5.03 & 2.63 & 9.54 & \multirow{2}{*}{0.469} \\
\hline & $\mathrm{B}$ & 33 & 5.62 & 1.82 & 5.72 & 1.82 & 9.35 & \\
\hline \multirow{2}{*}{ Fibrinogen $3(\mathrm{~g} / \mathrm{L})$} & $\mathrm{A}$ & 30 & 7.05 & 2.23 & 7.32 & 4.88 & 12.10 & \multirow{2}{*}{0.364} \\
\hline & $\mathrm{B}$ & 17 & 7.64 & 1.98 & 7.23 & 4.04 & 13.30 & \\
\hline
\end{tabular}


brinogen 1 and fibrinogen 3 parameters as predictors of the severity of AP was obtained. The percentage of successful prediction of moderate or severe AP based on this model was $76.9 \%$. Bearing in mind that a small number of factors have a significantly good diagnostic power (which was determined in ROC analysis), the idea was to use a linear combination of the selected factors as a diagnostic test. If we feed the linear combination from the binary logistic regression into ROC analysis, we obtain better results. First, a BINREG function is formed:

BINREG $=$ CRP* $(-0.007)+\mathrm{PCT}^{*}(-0.134)+$ D-dimer1*0.001 + D-dimer3*(-0.002) + Fibrinogen $1 * 0.429+$ Fibrinogen $3 * 0.165-1.989$

ROC analysis provided the results shown in Fig. 1.

A cut-off value of this function was 0.0112 , because the maximum sensitivity specificity product was achieved here. Patients with values on this function higher than or equal to the cut-off value had a mild form of AP (group A),

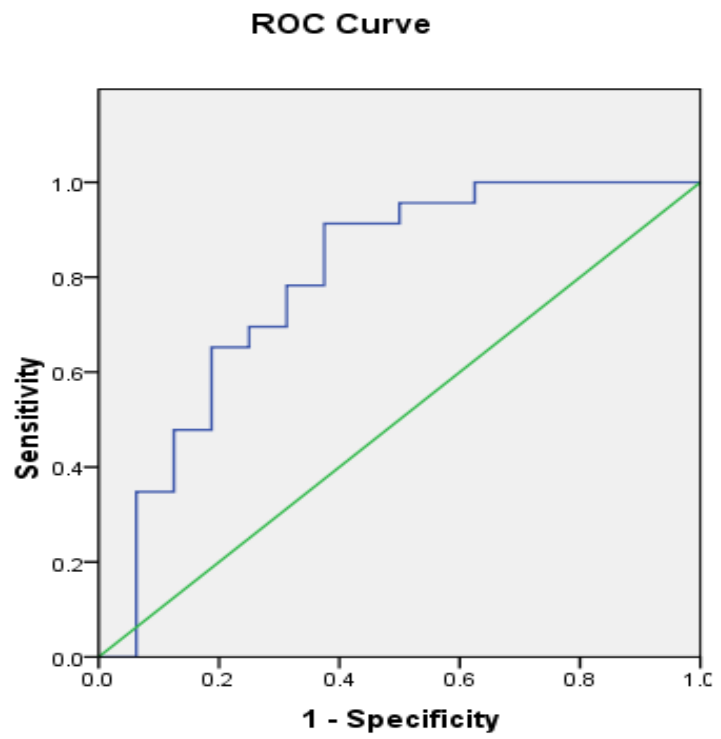

Figure 1. ROC curve of linear combination of CRP, PCT, D-dimer1, D-dimer3, Fibrinogen1 and Fibrinogen 3 from the logistic regression as diagnostic test between group $A$ and group $B$ and those with a value lower than 0.0112 had a moderate or severe form of AP (group B). In this way, accuracy of $79.5 \%$ of correct classifications was achieved. Using the linear combination from the binary logistic regression in ROC analysis, a diagnostic model with even better performance was obtained.

Using the t-test, a statistically significant difference between values of D-dimer 1 of the group II and III $(\mathrm{p}<0.05)$ was found. In the analysis of other parameters, the t-test did not show statistically significant differences.

Binary logistic regression which includes all the observed parameters gives a model which allows an accurate differentiation of the moderate (group II) and severe (group III) AP in the observed sample. The criterion for differentiating the severity is the function $\mathrm{pr}=\mathrm{y} /(1+\mathrm{y})$, where:

$\mathrm{y}=0.070 \mathrm{CRP}+0.965$ PCT +0.004 D-dimer1 + 0.002 D-dimer3 - 1.428 PT1 + 0.394 PT3 + 5.849 APTT1 - 9.599 APTT3 - 171.976 INR1 + 115.351 INR3 - 6.756 Fibrinogen1 - 4.887 Fibrinogen $3+314.552$

If the value of the function pr is lower than 0.5 , the patient will have a moderate form of AP; if $\mathrm{pr}$ is equal or above 0.5 , then the patient will have severe AP.

Using the Wald's stepwise forward method again, the second prediction model is obtained from PCT, Fibrinogen1 and Fibrinogen3. By analyzing patients diagnosed with severe AP, it can be concluded that $93.8 \%$ of the patients were correctly classified. Logics of the prediction is the same as in the previous model, with the only difference that $\mathrm{y}$ is calculated by the following formula:

$\mathrm{y}=0.150 \cdot \mathrm{PCT}-0.893 \cdot$ Fibrinogen $1-$ $0.404 \cdot$ Fibrinogen $3+4.425$

The cut-off value is $y=0.6052$. All patients with y higher than or equal to the cut-off value will have the severe form of AP. Using thus obtained diagnostics, $93.8 \%$ of patients were also classified correctly. 


\section{Discussion}

In AP, the release of proinflammatory mediators into the circulation can affect the coagulation process [6]. The released inflammatory mediators affect hemostasis by causing a fast activation of coagulation, with consequential global or partial exhaustion of physiological anticoagulation systems. Coagulation disorders in AP can be found ranging from localized intravascular thrombosis to disseminated intravascular coagulation (DIC) [13]. Because of a close connection between these mechanisms, during our study, we determined values of the inflammatory parameters CRP and PCT, as well as the coagulation parameters. Mean values of CRP and PCT, determined on admission, were higher than normal values. A statistically significant difference in CRP values between the group with mild and the group with moderate or severe AP was found, while there was no statistically significant difference in PCT values between these groups of patients. Melzi d'Eril et al. obtained similar results in a study that included 31 patients with AP. The authors did not verify statistically significant difference in PCT between the group with mild AP and the group with severe AP. They concluded that such a result was probably in agreement with the absence of infective complications in their patients. However, they did find a significant difference in CRP values between these two groups of patients and concluded that CRP was a better marker for assessment of the AP severity than PCT [14]. Different studies showed contradictory results, so the clinical significance of PCT in AP is still debatable $[15,16,17]$. In a multicentric study, Rau et al. concluded that PCT did not allow prediction of pancreas infection; however, it was a reliable parameter for predicting clinically significant infected necrosis which would be complicated by MODS. The authors concluded that PCT was a reliable parameter for assessment of a general prognosis during the course of severe AP. A CRP value analysis in this study did not show significant differences between patients with mild and moderate or severe AP [18]. The study by Pezzilli et al. on 31 patients with AP established the value of serum PCT within the first 24 hours of the onset of symptoms. This study found that the effectiveness of PCT in distinguishing mild and other AP was determined by the percentage of correct classifications of $58.2 \%$ for the cut off values between 0.252 to $0.255 \mathrm{ng} / \mathrm{ml}$. The effectiveness of PCT determined in this way is lower than the effectiveness of CRP indicated by the same study [19].

Serum C-reactive protein is frequently used for assessing the severity of AP. Opinions about its relevance in the early prediction of severe AP are divided. Tenner et al. reported that CRP has no significant predictive role in assessing the severity of AP in the first 72 hours after admission [20]. However, recent reports indicate that CRP determined in the first 24 hours after admission has a good predictive value for intrahospital mortality in AP. Cardoso et al. in their study emphasized the role of CRP in the early assessment of the severity of AP, especially in the case of a mild form of the disease [21].

In our study, we determined the CRP value on admission. The mean value of CRP was 12 times higher than the upper limit of the reference range. A statistically significant difference in CRP values was found between groups with mild and moderate or severe AP, i.e. patients from the group of moderate or severe AP had significantly higher values of CRP on admission. Coagulation disorders in AP can be manifested with different intensities, from occult to clinically manifested bleeding. Studies have shown that complications related to hemostatic disorders are some of the causes of such a high mortality rate among patients with AP. Despite the fact that numerous experimental proofs suggest an important role of the coagulation system in AP, studies concern- 
ing the clinical significance of parameters of the coagulation system for predicting complications due to pancreatitis are still very rare [22].

Values of DIC parameters and thrombin-antithrombin complex on admission to hospital are associated with poor prognosis of AP. A fourfold increase in D-dimer, which indicates the occurrence of fibrinolysis, appears to be a marker of complicated AP [23]. In cases of severe AP, patients who did not survive had significantly higher values of D-dimer than those who survived [24].

In our study, D-dimer proved to be a statistically significant parameter for determining severe AP on admission. We found statistically significant difference in D-dimer values between groups with moderate and severe AP, but well as significantly higher D-dimer values in patients with a severe form of the disease. The study by Badhal et al. showed high values of fibrinogen and D-dimer in AP [13]. This study did not find a statistically significant difference between values of PT and APPT in relation to the severity of AP. The authors conclude that coagulation disorders in AP, due to the acute inflammatory response, resulted in potential fibrinolysis by activation and downregulation of natural anticoagulants. In a study of 36 patients with AP, Boskovic et al. found that D-dimer could be a simple clinical predictor of AP severity [25].

By isolating certain inflammatory biomarkers (CRP and PCT) and parameters of coagulation system (D-dimer 1, D-dimer 3, fibrinogen1, fibrinogen3, PT1, PT13, APTT1, APTT3, INR1 and INR3) we suggest a mathematical model based on which we can accurately assess the severity of AP.

\section{Conclusion}

Determining individual values of CRP, PCT and parameters of the coagulation system allows for the early detection of clinically severe forms of acute pancreatitis.
The use of ROC analysis with the introduced linear combination from the logistic regression yielded equally good or even better results in the assessment of the severity of AP with the combined use of the analyzed parameters. The combined analysis of biohumoral markers and coagulation parameters presented in the form of a mathematical formula can enable a more accurate, rapid, rational, and clinically available prediction of the severity of AP. In our circumstances, this would facilitate the choice of an adequate and timely therapy, as well as the selection of patients to be transported into larger centers, where there are conditions for multidisciplinary treatment and more comprehensive therapeutic procedures.

\section{Abbreviations}

CRP - C-Reactive protein

PCT - procalcitonin

PT - prothrombin time

APTT - partial thromboplastin time

INR - international normalized ratio

\section{Acknowledgements}

The authors would like to thank dr Lara Zebic BDS from Birmingham in England, for her helpful advice.

\section{References}

1. Do JH. Mechanism of Severe Acute Pancreatitis: focusing on development and progression. The Korean Journal of Pancreas and Biliary Tract 2015 Jul;20(3):11523. DOI: $10.15279 / \mathrm{kpba} .2015 .20 .3 .115$

2. Fiusa MML, Carvalho-Filho A, Annichino-Bizzacchi JM, De Paula EV: Causes and consequences of coagulation activation in sepsis: an evolutionary medicine perspective. BMC Med 2015 May; 13: 105. DOI: 10.1186/s12916-015-0327-2

3. Lisman T, Porte RJ. Activation and regulation of hemostasis in acute liver failure and acute pancreatitis. Semin Thromb Hemost. 2010;36:437-43. DOI: 10.1055/ s-0030-1254052

4. Yang CJ, Chen J, Phillips ARJ, Windsor JA, Petrov MS. Predictors of severe and critical acute pancre- 
atitis: A systematic review. Dig Liver Dis 2014 May; 46(5):446-51. DOI: 10.1016/j.dld.2014.01.158

5. Thandassery RB, Yadav TD, Dutta U, Appasani S, Singh K, Kochhar R. Prospective validation of a 4-category classification of acute pancreatitis severity. Pancreas 2013 Apr;42(3):392-6. DOI: 10.1097/ MPA.0b013e3182730d19

6. Granger J, Remick D. Acute pancreatitis: models, markers, and mediators. Shock 2005 Dec; 24(Suppl 1):45-51. DOI: 10.1097/01.shk.0000191413.94461.b0

7. Banks PA, Bollen TL, Dervenis C, Gooszen HG, Johnson CD, Sarr MG, et al. Classification of acute pancreatitis 2012: revision of the Atlanta classification and definitions by international consensus. Gut 2013; 62:102-11. DOI: 10.1136/gutjnl-2012-302779

8. Meher S, Mishra TS, Sasmal PK, Rath S, Sharma R, Rout B, Sahu MK. Role of Biomarkers in Diagnosis and Prognostic Evaluation of Acute Pancreatitis. J Biomark 2015; Article ID 519534, 13 pages.

9. Khanna AK, Meher S, Prakash S, Tiwary SK, Singh U, Srivastava A, et al. Comparison of Ranson, Glasgow, MOSS, SIRS, BISAP, APACHE-II, CTSI Scores, IL6, CRP, and procalcitonin in predicting severity, organ failure, pancreatic necrosis, and mortality in acute pancreatitis. HPB Surgery 2013; Article ID 367581, 10 pages.

10. Matull WR, Pereira SP, O’Donohue JW. Biochemical markers of acute pancreatitis J Clin Pathology 2006; 59(4):340-4. DOI: 10.1136/jcp.2002.002923

11. Bettina R, Schilling MK, Beger HG. Laboratory markers of severe acute pancreatitis. Dig Dis 2004;22:24757.

12. Bradley 3rd EL. A clinically based classification system for acute pancreatitis. Summary of the International Symposium on Acute Pancreatitis, Atlanta, Ga, September 11 through 13, 1992. Arch Surg 1993;128:58690. DOI: 10.1001/archsurg.1993.01420170122019

13. Badhal SS, Sharma S, Saraya A, Mukhopadhyay AK. Prognostic significance of D-dimer, natural anticoagulants and routine coagulation parameters in acute pancreatitis. Trop Gastroenterol 2012 Jul-Sep; 33(3):1939. DOI: $10.7869 / \operatorname{tg} .2012 .48$

14. Melzi D’Eril, GV, Merlini G, Finazzi S, Bosoni T, Barakat B, Pezzilli R. Procalcitonin is not a reliable marker for the assessment of severity in acute pancreatitis without infectious complications. Clin Chem 2000 Mar; 46(3):428-30.

15. Muller CA, Uhl W, Printzen G, Gloor B, Bischofberger H, Tcholakov O, Buchler MW. Role of procalcitonin and granulocyte colony stimulating factor in the early prediction of infected necrosis in severe acute pancreatitis. Gut 2000; 46(2):233-8. DOI: 10.1136/ gut.46.2.233

16. Frasquet J, Saez J, Trigo C, Martínez J, Such J, Pérez-Mateo M, et al. Early measurement of procalcitonin does not predict severity in patients with acute pancreatitis. Br J Surg 2003 Sep; 90(9):1129-30. DOI: 10.1002/bjs.4172

17. Modrau IS, Floyd AK, Thorlacius-Ussing O. The clinical value of procalcitonin in early assessment of acute pancreatitis. Am J Gastroenterol 2005 Jul; 100(7):15937. DOI: $10.1111 / \mathrm{j} .1572-0241.2005 .41456 . \mathrm{x}$

18. Rau BM, Kemppainen EA, Gumbs AA, Büchler MW, Wegscheider K, Bassi C, et al. Early Assessment of Pancreatic Infections and Overall Prognosis in Severe Acute Pancreatitis by Procalcitonin: A Prospective International Multicenter Study. Ann Surg 2007 May; 245(5):745-54. DOI: 10.1097/01. sla.0000252443.22360.46

19. Pezzilli R, D’Eril GVM, Morselli-Labate AM, Merlini G, Barakat B, Bosoni $\mathrm{T}$ et al. Serum amyloid A, procalcitonin, and C-reactive protein in early assessment of severity of acute pancreatitis. Dig Dis Sci 2000 Jun; 45(6):1072-8. DOI: 10.1023/A:1005525329939

20. Tenner S, Baillie J, DeWitt J, Swaroop Vege S. American college of gastroenterology guideline: management of acute pancreatitis. Am J Gastroenterol 2013 Sep; 108(9):1400-15. DOI: 10.1038/ajg.2013.218

21. Cardoso FS, Ricardo LB, Oliveira AM, Horta DV, Papoila AL, Deus JR et al. C-reactive Protein at 24 Hours after Hospital Admission May Have Relevant Prognostic Accuracy in Acute Pancreatitis: A Retrospective Cohort Study. GE Port J Gastroenterol. 2015; 22(5):198203. DOI: 10.1016/j.jpge.2015.03.002

22. Gould T, Mai S, Liaw PC. Coagulation Abnormalities in Acute Pancreatitis, In: Rodrigo L, Editor. Pancreatitis - Treatment and Complications. InTech: 2012 p. 59-72. DOI: $10.5772 / 30169$

23. Salomone T, Tosi P, Palareti G, Tomassetti P, Migliori M, Guariento A, et al. Coagulative disorders in human acute pancreatitis: role for the D-dimer. Pancreas 2003 Mar; 26(2):111-6. DOI: 10.1097/00006676200303000-00003

24. Radenkovic D, Bajec D, Ivancevic N, Milic N, Bumbasirevic V, Jeremic V, et al. D-dimer in acute pancreatitis: a new approach for an early assessment of organ failure. Pancreas 2009 Aug; 38(6):655-60. DOI: 10.1097/MPA.0b013e3181a66860

25. Boskovic A, Pasic S, Soldatovic I, Milinic N, Stankovic I. The role of D-dimer in prediction of the course and outcome in pediatric acute pancreatitis. Pancreatology 2014 Sep-Oct; 14(5):330-4. DOI: 10.1016/j. pan.2014.07.015 